\title{
A Cyber-Physical System for Surface Roughness Monitoring in End-Milling
}

\author{
Uroš Župerl* - Franci Čuš \\ University of Maribor, Faculty of Mechanical Engineering, Slovenia
}

The main focus of this paper is to describe the structure of the cyber-physical machining system developed for on-line surface roughness monitoring via cutting chip size control in end-milling. The two level cyber- physical machining system is realized by connecting the computing resources in the developed cloud machining platform to the machine tool with its smart sensor system. The smart optical sensor system is developed to acquire and transfer in real time the values of the cutting chips sizes to the cloud level. The cloud based machining platform with the developed internet of things applications is employed to perform instant surface roughness monitoring and cutting chip size control based on advanced sensor signal processing, edge computing, process feature extraction, machine learning, process modeling, data analyzing and cognitive corrective process control acting. These actions are performed as cloud services. A cloud application with an adaptive neural inference system is applied to model and on-line predict surface roughness based on the determined cutting chip size. Based on the inprocess predictions, a novel application for cognitive corrective control acting is employed to control the cutting chip size by modifying the machining parameters and consequently keeping surface roughness constant. The results of the machining experiment are presented to demonstrate that this proposed system where the cloud computing resources and services are linked with the machine tool is feasible and could be implemented to monitor surface roughness during milling operation.

Keywords: machining, end milling, chip size control, surface roughness, monitoring

Highlights

- Cyber-physical machining system is developed to realize end-milling process monitoring.

- A new way for controlling the chip size and monitoring the surface roughness is developed.

- An adaptive neural inference system is applied to in-process predict the surface roughness.

- A visual system is employed to detect the cutting chip size.

- Machining experiment for testing the cloud based machining platform is provided.

\section{INTRODUCTION}

Modern machining systems dealing with variety of products in small series demand real-time monitoring abilities that are adaptive to the sudden changes of cutting conditions. The modern computer numerical control (CNC) controllers and smart sensors are now efficient and intelligent enough to communicate with devices and process the raw data during machining. Internet of Things (IoT) and cloud manufacturing technologies provide an efficient environment for integrating and connecting knowledge, software, computing and physical parts of distributed machining equipment together. These new cloud technologies can be employed for efficient machining process monitoring and control applications. They can be used to connect machine tool and smart sensors together with cloud based monitoring capabilities via the internet networking infrastructure. Such systems that connect the cyber-world of computing, service resources and communications in the cloud with the physical devices are referred to as cyber-physical systems (CPSs) [1]. The cloud based smart monitoring systems may consequentially increase productivity, reduce machining cost and improve surface quality by efficient system adaptation.

Self-monitoring, analysis, and reporting technology (Smart or S.M.A.R.T) is a monitoring system with the aim to predict or anticipate the potential process problems or failures. It includes sensing, actuation and control functions in order to analyze a process and perform actions based on the available data in an adaptive manner. The smartness of the system is often attributed to autonomous operation based on closed loop control and networking capabilities [1]. Recently, a few smart machining systems have been designed to carry out adaptive control of machine tools. Wang et al. [2] presented a concept and innovative use of adaptive smart machining based on using constant cutting force and a smart cutting tool, developed by authors. Teti et al. [3] reviewed advanced monitoring achievements in machining operations, from sensorial perception and feature extraction to cognitive decision making and action.

Nowadays, surface finish monitoring control becomes increasingly important for the smooth operation of modern machine tools. 
Recently, the research efforts to improve surface finish of machined parts have been oriented towards developing of advanced surface finish control systems which contain reliable in-process models that can efficiently predict surface roughness. A statistical model to correlate surface roughness and cutting force in end-milling operations is employed in a research of Chen and Huang [4]. Abburi and Dixit [5] apply machining parameters and two cutting force components for online prediction of surface finish. Rao et al. [6] use singular spectrum analysis to decompose the vibration signals for in-process prediction of surface roughness in turning. Oborski [7] investigates time series analysis of vibration acceleration signals for real-time prediction of surface roughness. Marinescu and Axinte [8] and Axinte et al. [9] use AE signals and cutting force data to detect surface anomalies in milling of aerospace materials. Some systems of surface roughness control have been developed for turning operations, with only a few for end milling operations. Yang et al. [10] developed a fuzzy-nets-based in-process surface roughness adaptive system in end milling. The developed system has the ability to change cutting parameters to produce a high-quality product in-process using a fuzzy-nets adaptive feed rate control system. Zhang and Chen [11] employed a multiple linear regression algorithm to establish an in-process surface roughness evaluation system and an in-process adaptive parameter control (IAPC) system. Zuperl and Cus [12] employ in their model reference adaptive control system (MRAC) a dynamometer as an in-process sensor to indirectly measure and control the surface roughness in milling. The same technique was also applied to the turning process [13]. To address the challenges caused by indirect measuring and control of surface roughness, a new cyber-physical machining system (CPMS) for controlling the cutting chip size and monitoring the surface roughness through internet applications is presented in this paper. In this approach a visual measuring system acquires and transmits the signals of the cutting chip size through the internet to a cloud-based application for control decision making and acting. The latter is responsible for adjusting the optimal cutting parameters.

The paper is organized as follows: In the second section of the paper a CPMS for surface roughness monitoring is presented. Section 3 describes architecture of cloud based machining platform with detailed descriptions of integrated Internet of Things (IoT) applications. Section 4 presents physical resources, experimental results and discussion. Finally, section 5 concludes the paper.

\section{CYBER-PHYSICAL MACHINING SYSTEM FOR ROUGHNESS MONITORING}

The goal of this research is to develop a CPMS for surface roughness monitoring and control during milling. The proposed system is intended for machining of difficult to cut materials, where small spiral, arc or short coma chips were produced without high pressure cooling. These chip shapes are typically produced when machining workpiece materials with high hardness and low thermal conductivity, such as titanium alloys, nickel alloys and hardened steels (AISI D2 steel of 62 HRC). The CPMS adapts online the machining parameters in order to maintain the cutting chip size constant and therefore, to attain the desired value of the machined surface finish. The CPMS serves as a cloud surface roughness monitoring system and is based on the structure shown in Fig. 1. The proposed system is built in two levels. The lower level is a factory or machine level. This level incorporates physical assets (machine tool, optical vision system) and software resources (data acquisition) located in the machining system. The physical resources are connected to the computing resources (monitoring, analysing, data visualization, decision making) in the IoT machining platform located in the upper, cloud level. By linking these two levels a complex cyber-physical machining system is formed. During machining, the two level system collects the signals from vision system and employs them for surface roughness monitoring and feedback control actions. The sensor signal pre-processing is executed on the local machine level. The sensor data are collected, reduced, transformed into data packages and transferred to the IoT machining platform over the local area network (LAN). The cloud machining platform accepts the signal packages, processes the signals, saves the data, assesses the cutting chip size, calculates the chip size average, predicts the machined $R a$, visualizes the machining data and selects the control actions in order to monitor the machining process. After all the listed tasks have been performed, the IoT platform sends back the control actions/ decisions signals with the graphical representation of monitoring results to the lower machine level (local communication terminal). The executed control actions with alerts and explanations are visualized for machine tool operator on the control panel of the local terminal. The local terminal serves as a communication link between $\mathrm{CNC}$ unit, machining IoT platform and machine tool operator. 


\section{CLOUD BASED MACHINING PLATFORM}

The IoT machining platform serves as the monitoring platform by using the advantages of the cloud capabilities such as outsourcing signal processing activities, graphical data visualisation, process value predicting and cognitive control decision making. The IoT machining platform is connected to the private machining cloud LAN.

The machining platform consists of seven parts. The first part is an IoT gateway which implements security, enables communication bridging, collects the enormous amount of sensorial data and filters data volume. These are edge computing activities. The IoT gateway collects the pre-processed data and reduces it to the amount needed by the monitoring process. The data is sorted according to the time filter. The data ends up in a machine tool business system, where it is combined with existing machine tool system data such as meta data or transaction data (part 2). In the third part, further signal processing is carried out in order to extract the relevant features for surface roughness monitoring process. For this purpose, an IoT application is built to detect the cutting chip size as relevant feature from the acquired sensor data. The fourth application is used to estimate the surface roughness $R a$ through ANFIS based prediction approach. The fifth application calculates the chip size. The sixth application is used for cognitive control actions such as process parameters corrections. The final application performs the graphical data visualization.

\subsection{Application for Cutting Chip Size Acquiring}

A sensor signal feature (cutting chip size) which provides the adequate information about the machined $R a$ is extracted from the pre-processed sensorial data in this IoT application.

An algorithm for cutting chip size acquiring was developed In Labview and is described in the research [14]. The five main steps (a-e) of the algorithm are

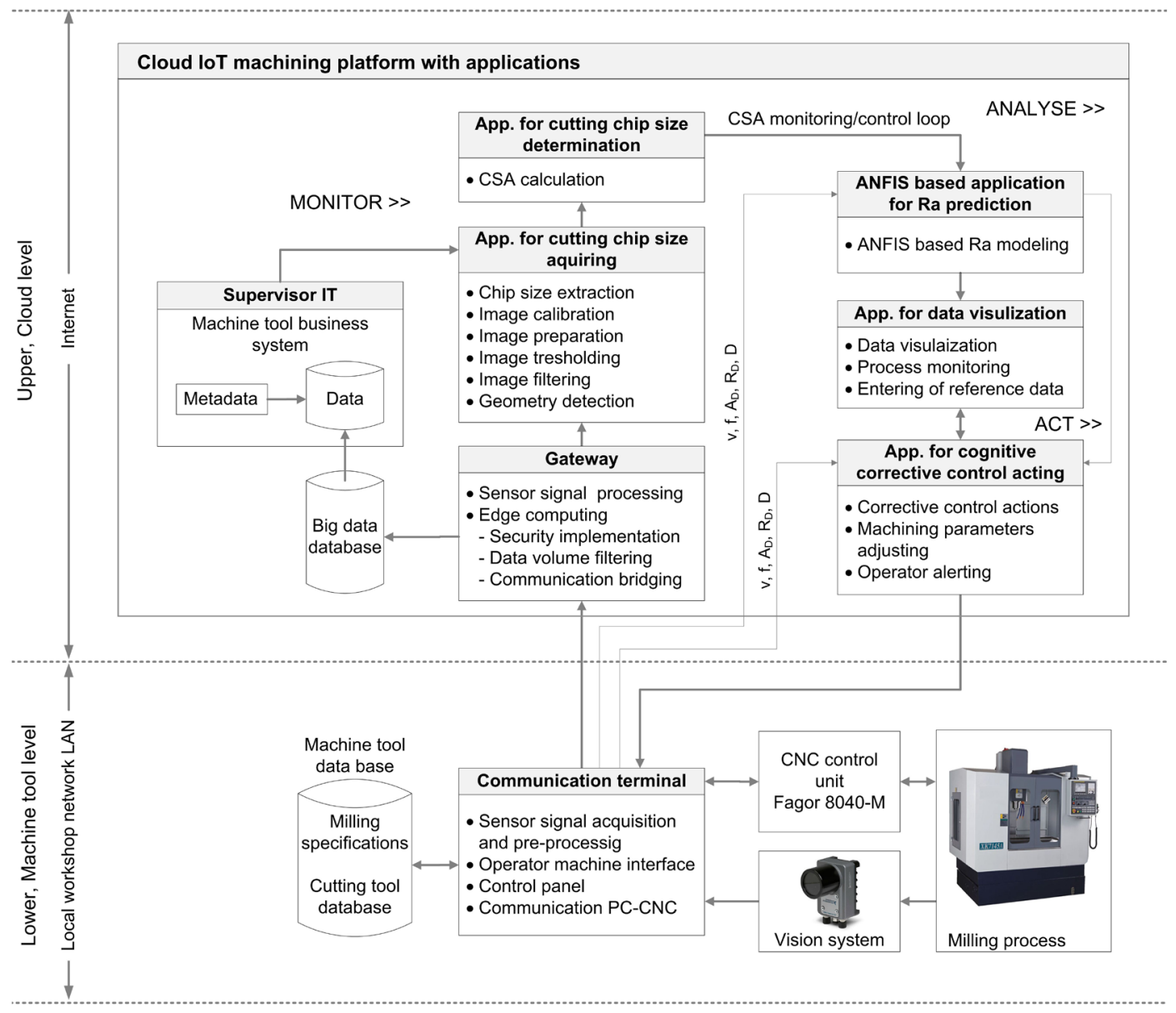

Fig. 1. The basic structure of the two level CPMS 


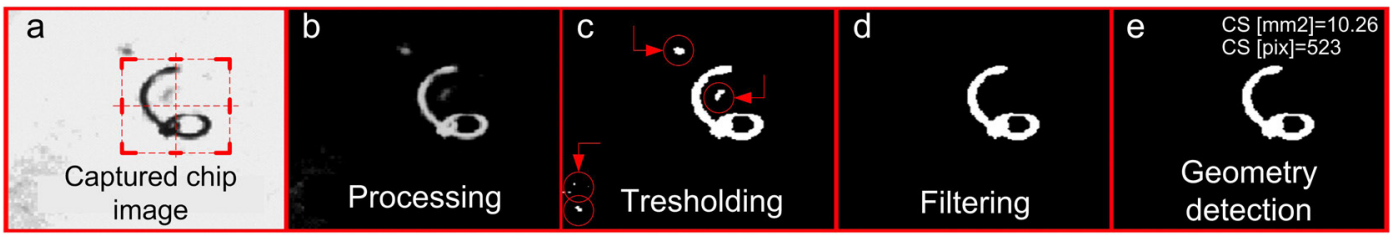

Fig. 2. Steps of the cutting chip size acquiring algorithm

presented in Fig. 2. Fig. 2 shows the result of the chip image capturing, processing, tresholding, filtering, geometry detection and variable determining process. Data transition between the IoT applications is accomplished via 6 system variables which contain data of the acquired chips for particular iteration of detection.

\subsection{Application for Cutting Chip Size Determination}

The optical sensor detects a great number of chips with diverse sizes; hence the determination of the chip size average (CSA) is required. The CSA is determined based on shift registers and system variables for every 25 consecutive detected cutting chips. The number of samples for calculating of the CSA is arbitrary defined in the range from 2 to 70 on the control panel for $R a$ monitoring and CSA control (Fig. 3).

The vision system is able to precisely detect chip sizes in the range from $0.05 \mathrm{~mm}^{2}\left(C S_{\min }\right)$ to $250 \mathrm{~mm}^{2}$ $\left(C S_{\max }\right)$.

The CSA actually represents average surface of the chips projections in $\mathrm{mm}^{2}$ on the plane perpendicular to the optical axis of the vision system. Therefore, the CSA depends on the shape and size of the chips. The shape of the chip depends on the chip thickness ratio, cutting speed, feed rate, depth of cut, workpiece material, workpiece surface quality, lubricant, tool material and tool geometry [15].

Many performed analyses of machining of NiCrMo heat-treated alloy steel, showed that there are relationships between the chip size and cutting conditions [16]. Therefore, in future research, it would be sensible to determine with intelligent modelling methods (ANFIS or genetic programming) the correlation between the CSA and cutting parameters.

The tool wear causes changes in tool tip geometry and changes on the tool flank surface. Both have significant influence on the chip forming process and chip geometry [17]. The changes in the geometry of the machined chips with the progress of tool wear for given cutting conditions were investigated in the study of Dargusch et al. [17]. Significant change in chip geometry was observed in the chips which were produced first with the new tool and then with the worn tool. It was also found that the chip geometry changes with an increase in the volume of material removed, which is believed to be the result of progressive changes in the tool geometry and an increase in cutting temperature due to tool wear [18]. Since the CSA is correlated with geometry and size of the chip, the tool wear has a significant impact on the CSA.

The CSA value is the input to the ANFIS based application for $R a$ prediction, the application for data visualization and the application for cognitive corrective control acting. The CSA value is connected with the indicator on the human machine interface.

\subsection{ANFIS Based Application for $R a$ Prediction}

The second platform application is devoted to online predict the $R a$ by automatically finding the connections between machining parameters, $D, C S A$ and $R a$. The testing error of the developed model was found to be less than $4 \%$. The detail adaptive neural fuzzy inference system (ANFIS) architecture employed for $R a$ prediction procedure is shown in Fig. 4.

The ANFIS model is developed based on training. The internal structure of the finished model is fixed, thus it acts as deterministic model where the input data vector always returns a specific output $R a$ value. The performance of ANFIS prediction mainly depends on the completeness of the input data. It is, therefore, necessary to evaluate the ANFIS performance via various calibration samples in order to estimate the model uncertainty caused by changes in input data.

In order to calculate the uncertainty in the $R a$ prediction, a Monte Carlo (MC) simulation is performed by running the $R a$ model many times either varying randomly the input data (training and validation set) or the training parameters (neural weights), or a combination of both.

The ANFIS system has been incorporated into the MC sampling structure as prescribed by Marcé et al. [19]. Three main steps are performed in prescribed procedure: multiple constituting a random vector 


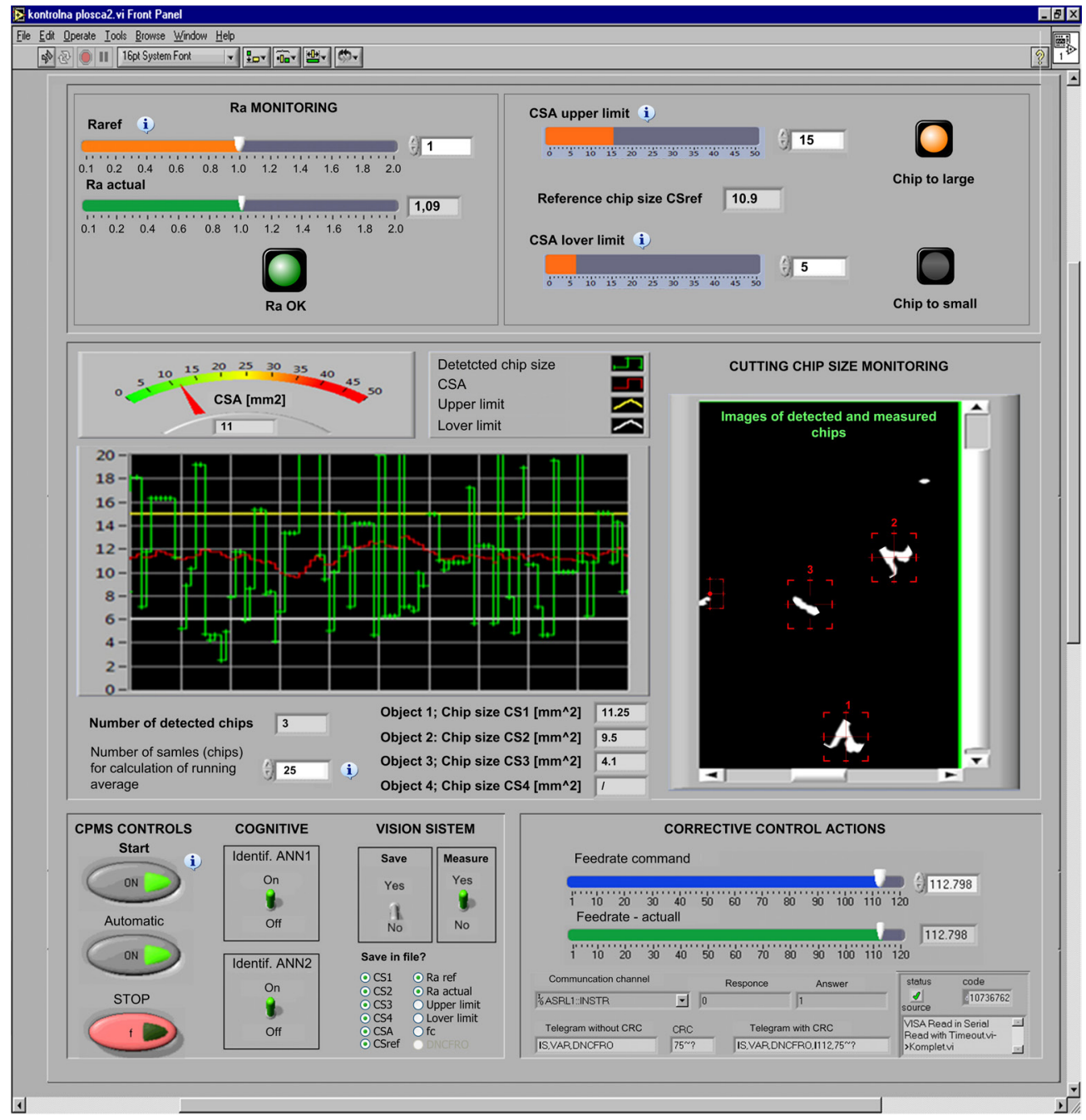

Fig. 3. Graphical user interface for Ra monitoring and CSA control

of parameters from their probability distributions, assessing the output $(R a)$, computing the statistics of the output and plotting the distributions of resulting statistical performances. The uncertainty of the model estimations of the measured and predicted $R a$ during the training and testing process has been determined by estimating the confidence intervals of simulation outputs. The $95 \%$ confidence intervals are determined by finding the $2.5^{\text {th }}$ and $97.5^{\text {th }}$ percentiles of the associated distribution of the simulation results.

The results of the MC simulations indicate that almost all of predicted $R a$ values are within the $95 \%$ confidence intervals. The simulation results reveal that the ANFIS prediction model sometimes underestimated the values of $R a$ during machining at small feed rates and at small chip sizes. However, just a $1.8 \%$ of the predicted-observed values were found above the upper bound. The extent of these intervals with respect to the observed values was $92 \%$.

The low model accuracy at small feed rates and small chip sizes is probably due to the missing values in the training data set which created the model too sensitive. The second reason is the inability to produce appropriate fuzzy rules based on training data.

The investigation of the uncertainty attributed to the ANFIS structure would require large computational effort; therefore it is not examined in this study. It is also assumed that the model structure and the input data are correct.

The uncertainty of the predicted results, obtained from the proposed methodology, can be characterized as small, hence it can be concluded that the ANFIS adequately predicted the $R a$. The average percentage prediction error of the trained ANFIS model is less than $3.7 \%$. 
The monitoring process is based on visual sensor signal acquisition of the CS. Sensor signals are sent to the gateway on the cloud machining platform. The gateway sequentially buffers the sensor signal parts, which are then transported to the application for the extraction of the relevant cutting chip features by using wavelet packet transform method. The extracted features are fed into the application to determine the CSA. The CSA together with the cutting conditions from the local communication terminal are fed into the ANFIS system for $R a$ prediction. The output from the ANFIS is $R a$ in micrometers. The $R a$ monitoring is performed twice per second. When the predicted $R a$ exceeds the desired surface roughness (Raref), the monitoring output is sent to the application for cognitive corrective control acting. The latter alerts the machine tool operator via the application for visualisation or sends the corrective control commands to $\mathrm{CNC}$ control in order to adjust the machining parameters via DNC control functions. The application for data visualisation visualises the alert with the indicators that the upper $R a$ boundary has been exceeded.

\subsection{Application for Data Visualization}

This application is built as a human machine interface in order to visualize a large amount of captured data from the sensor. It also serves as a control panel for $R a$ monitoring and CSA control system. Fig. 4 shows the graphical user interface (GUI) of the developed application, built in Labview. The input to the application is the reference $R a$ or the range of the CSA which is entered by two graphical sliders in an upper right part of the GUI. Three warning indicators inform the user about the exceeded value of the machined $R a$ and unsuitable cutting chip size. The centre part of the GUI displays the acquired cutting chip images with the diagram of corresponding $C S A$ values and the allowed chip size range. The GUI is projected on the local communication terminal.

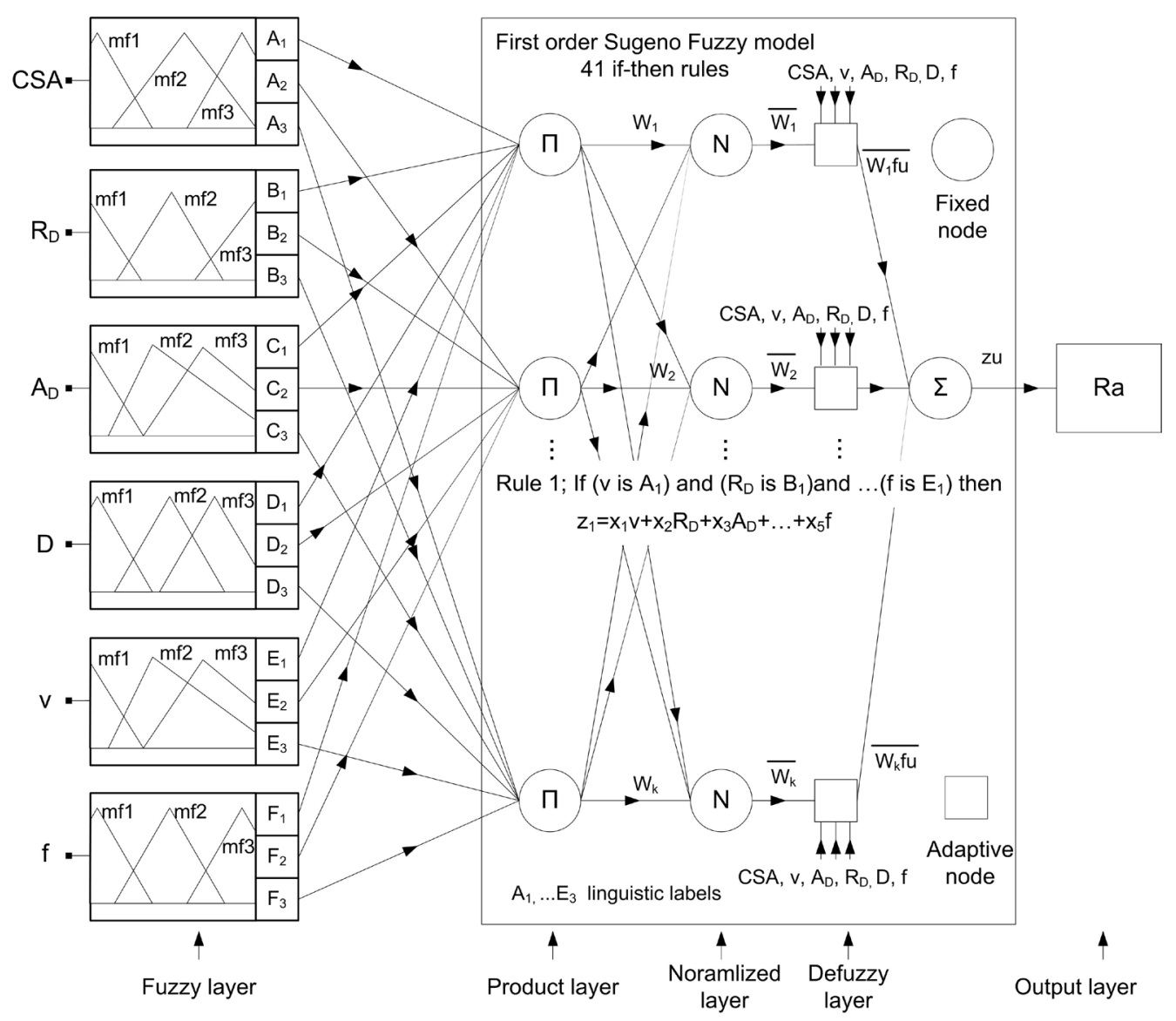

Fig. 4. Structure of ANFIS based Ra predicting

(multi-input Sugeno fuzzy model, generated rules, multi membership functions for each input) 


\subsection{Application for Cognitive Corrective Control Acting}

When the ANFIS based application predicts the $R a$ and the user enters the $R a_{\text {ref }}$, the cognitive corrective control actions are taken in this application. Application is built as a cognitive control system and has adaptive process parameter adjustment capability. If the significant change of the CSA is identified, the control actions are taken within $10 \mathrm{~ms}$ after the occurrence. Three steps are required to execute a control action: In step one, the reference module A1 predicts the CSref. The A1 is the inverse model of the model located in the cloud ANFIS based application for $R a$ prediction. In step 2, the feed rate adaptation is performed. During this stage, the cognitive control system adjusts the feed rate values in order to minimize the difference between CSref and CSA. Finally in the last step the $f$ value is sent to the application for data visualization and to the local communication terminal, where the feed rate command telegram is built and forwarded to the $\mathrm{CNC}$ control unit.

Fig. 5 shows the block diagram of the application for cognitive corrective control acting.

The principal control acting principle is based on a cognitive neural control system (CNCS). Its general structure consists of two elements and is depicted in Fig. 5.

The first part of the CNCS is a conventional control loop known as external feedback. The second, fundamental part is a sub-system of two connected artificial neural networks (ANN) with self learning capabilities. The loop connected with neural network 1 (ANN1) works as an internal feedback loop which is faster than the external feedback loop due to sensory delays.

The ANN1 monitors the process input $f$ and output CSA and learns the process dynamics. When trained on-line, it can precisely predict the process output $C S A^{*}$. It operates as the cognitive process dynamics identifier. The second artificial neural network (ANN2) learns the process inverse dynamics and monitors the $f$ and the CSref. After on-line training, it can predict appropriate $f^{*}$ based on received input command $C S r e f . f^{*}$ and $C S A *$ are the predicted process input and output by the two neural networks.

The CNCS acts as follows. The external feedback which assures a conventional feedback signal to control the milling is efficient particularly in the training phase. In this phase, the ANN1 learns the process dynamics and the ANN-2 learns the inverse

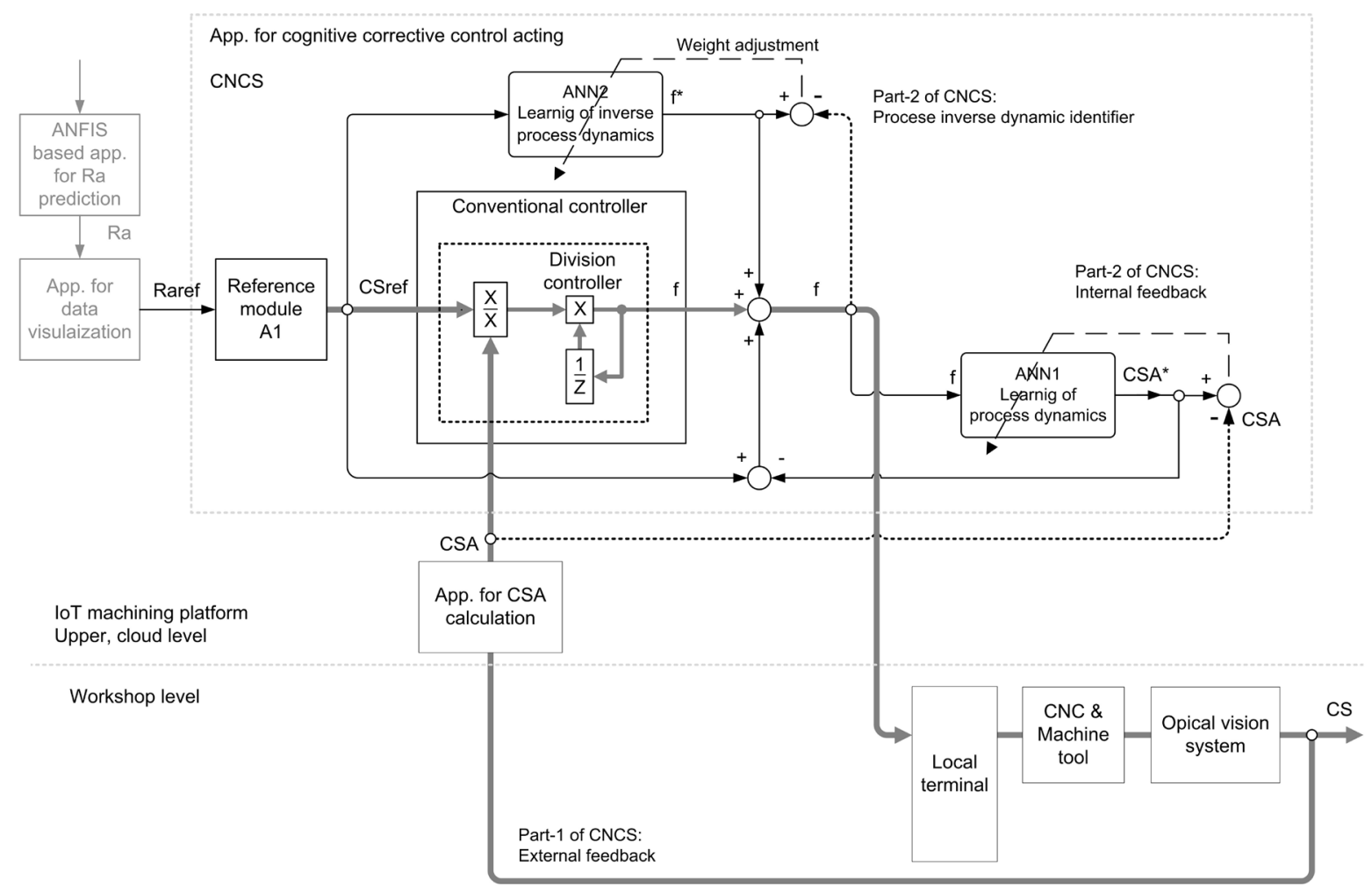

Fig. 5. General block diagram of the neural control system 
dynamics. As training progresses, the internal feedback progressively prevails over the external feedback. After a certain learning stage, the ANN2 will completely substitute the external feedback control. The result is that the milling process is controlled above all by the feedforward controller. To automatically learn the process dynamics and inverse dynamics in the CNCS, two four-layer feedforward ANN were employed. The architecture of these two ANNs is described in the work of Zuperl and Cus [12].

The employed conventional controller is very well known division controller which is very easy to implement and has fast response. The control algorithm determines the new feed rate corrective command based on previous command and the quotient between the CSref and the CSA.

\section{LOCAL COMMUNICATION TERMINAL}

The communication terminal serves as an interface for the local machine tool operator and as a communication link between $\mathrm{CNC}$ unit and the gateway on the cloud machining IoT platform. The local terminal visualizes the condition of machining process, displays the results of cloud machining platform monitoring, shows the performed control commands and displays the alerts. The terminal is also employed to pre-process the optical sensor signals, to transfer the sensor signals to the cloud platform and to send the control telegrams to the CNC control unit. During signal pre-processing, thousand of information bits from the optical sensor are collected and reduced by removing the transient conditions. The pre-processed signals are then transmitted via hyper text transfer method to the cloud machining platform in order to perform the surface roughness monitoring process.

\section{EXPERIMENTAL DEMONSTRATION}

To test the feasibility of the cloud based machining platform for monitoring of end-milling, one successful cutting experiment with variable milling depth has been carried out, in which the axial depth of cutting is varying between $4.75 \mathrm{~mm}$ and $9.5 \mathrm{~mm}$.

The workpiece material is a $16 \mathrm{MnCr} 5$ steel. The shape of the workpiece consists of two steps and two ramp parts, as shown in Fig. 6. The machining of these four parts of the workpiece has been performed in order to evaluate the stability and robustness of the CNCS to control the CSA. The cutting experiment has been carried out using the CNC machine tool Heller equipped with an optical vision system. A vision system consisting of a high speed smart camera NI $1772 \mathrm{C}$ was used to acquire useful information for $\mathrm{Ra}$ monitoring and CSA control from the cutting chip images. The maximum output is 64.995 frames per second (fps). The data are transferred to the cummunication terminal via giga ethernet cable. A ball-end milling tool of $16 \mathrm{~mm}$ diameter was used. After machining, the machined $R a$ was examined offline by 7061 MarSurf PS1 surface roughness tester. The tester has $8 \mathrm{~nm}$ resolution at $0.09 \mathrm{~mm}$ vertical range. The objective of the cutting experiment is to keep the $R a$ constant by minimizing the error between the CSref and process output CSA. The initial Raref was chosen equal to $1.9 \mu \mathrm{m}$ and the permitted control error was set to be less than $5 \%$. Based on the Raref, the CSref was set to $2.9 \mathrm{~mm}^{2}$ according to the prediction module A1. The spindle speed $(n)$ is set to $985 \mathrm{rpm}$, and the radial depth of cut $(R D)$ is set to $8 \mathrm{~mm}$. To achieve the $R a$ of $1.7 \mu \mathrm{m}$, the $C S A$ must be fixed at $2.9 \mathrm{~mm}^{2}$ and the feed rate must be below $835 \mathrm{~mm} / \mathrm{min}$. The starting feed rate command was set to $835 \mathrm{~mm} / \mathrm{min}$ with allowable adjusting rate in the range form $400 \mathrm{~mm} / \mathrm{min}$ to 1050 $\mathrm{mm} / \mathrm{min}$. The cutting experiment was started first without and then with the CNCS control. The result of the cutting experiment is presented in Fig. 6. The cutting experiment started with conventional cutting of the ramp part of the workpiece (0 mm to $40 \mathrm{~mm}$ ). The feed rate was kept constant. Without adjustment of feed rate, the $C S A$ changed from $13.5 \mathrm{~mm}^{2}$ to 8.5 $\mathrm{mm}^{2}$ and the $R a$ decreased from $2.02 \mu \mathrm{m}$ to $1.88 \mu \mathrm{m}$. Fig. 6a shows the response signal of the $R a$. Fig. $6 f$ presents the CSA response. The control action was employed at $t=2 \mathrm{~s}$ (position B). In the ramp part (45 $\mathrm{mm}$ to $70 \mathrm{~mm}$ ), the feed rate was adapted according to the value of the CSA. Firstly, the CNCS reduced the feed rate to $665 \mathrm{~mm} / \mathrm{min}$, as shown in Fig. 6i. As the $A_{D}$ decreased continuously, the feed rates increased accordingly and thus maintained the $R a$ at $1.7 \mu \mathrm{m}$. During cutting of the ramp part (segment A-B), the $C S A$ is kept constant so that the Raref can be attained. Fig. $6 \mathrm{f}$ and $\mathrm{g}$ show the $C S A$ variations without and with control action. The results indicate that the CSA control system operated well and had advantage over non-control. When, at the position $\mathrm{C}(L=70 \mathrm{~mm})$, the tool partially run into a step, a high peak of the CSA appeared and the $f$ decreased suddenly to keep the $R a$ at $1.7 \mu \mathrm{m}$. The CNCS was capable to compensate the induced perturbation. Fig. $6 \mathrm{c}$ shows the $R a$ response. Fig. $6 \mathrm{~m}$ shows the corresponding $\mathrm{f}$ response. The feed rate dropped to the value between $460 \mathrm{~mm} / \mathrm{min}$ and $510 \mathrm{~mm} / \mathrm{min}$ to maintain the $C S A$ average at 2.95 $\mathrm{mm}^{2}$. At the tool engagement, the maximum CSA 


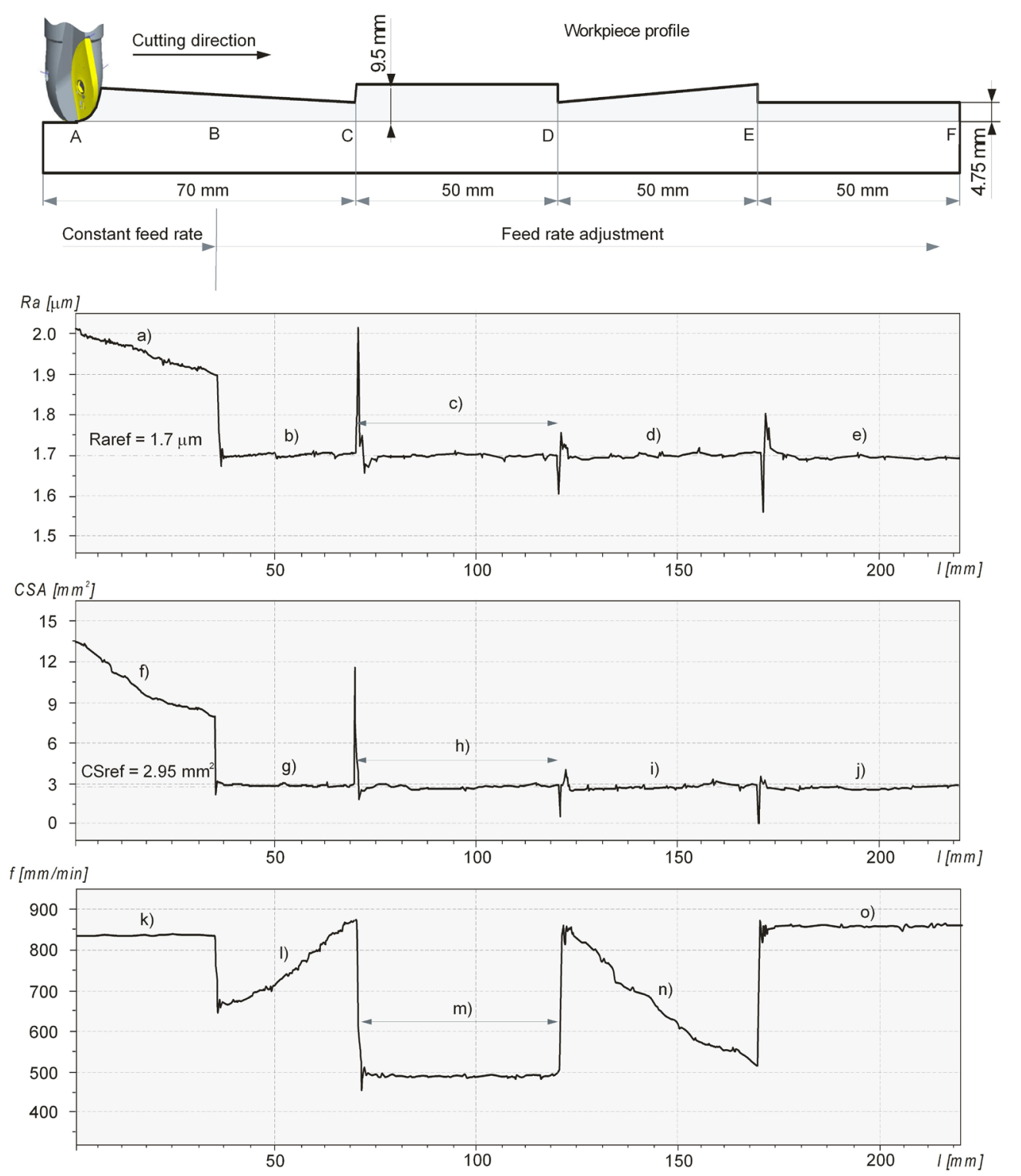

Fig. 6. Cutting experimental results of Ra, CSA and feed rate control command responses to the variable axial depth of cutting

was $11.6 \mathrm{~mm}^{2}$ and during the pass it was $2.95 \mathrm{~mm}^{2}$. In the range of step part ( $70 \mathrm{~mm}$ to $120 \mathrm{~mm})$ where $\mathrm{AD}$ is maximal, the feed rate command was almost constant. Fig. 6h shows the response of the CSA. At the position $\mathrm{D}(L=120 \mathrm{~mm})$ where the $A_{D}$ decreased, $\mathrm{f}$ increased suddenly to keep the CSA at the desired level. When the cutter encountered the slope, the feed rate started to decrease. In the ramp part (position E, $L=170 \mathrm{~mm}$ ), where the $A_{D}$ fell, $f$ increased suddenly. When the tool is out of the step, the feed rate control signal stabilized at $857 \mathrm{~mm} / \mathrm{m}$. In the range of step part (170 mm to $230 \mathrm{~mm}$ ) where the $A_{D}$ is $4.75 \mathrm{~mm}$, the feed rate command was nearly constant after the transitional stage.

During the cutting experiment the CNCS manages to return the CSA to the reference chip size within $0.48 \mathrm{~s}$. The CPMS performs the $R a$ monitoring loop every $0.07 \mathrm{~s}$.

The cutting experiment outlined that the $R a$ is kept constant by regulating the CSA.

\section{CONCLUSIONS}

In this paper, a cyber-physical machining system CPMS has been employed with the aim of monitoring 
the surface roughness by controlling the cutting chip size. The two layered complex CPMS was realized by integrating the cloud based IoT machining platform for process control to the machine tool with the visual system. The machining platform with the IoT applications offers instant on-line chip size and surface roughness monitoring based on visual sensorial data acquired at the workshop level and the ANFIS algorithm. Based on the cloud monitoring results, the cognitive neural control system CNCS determines the required corrective feed rate adaptation. The local communication terminal in connection with the $\mathrm{CNC}$ unit executes the cloud based corrective control actions.

The important advantage of the IoT machining platform is the increased distributed analytic and data storage/processing capacity which significantly improves the surface roughness monitoring efficiency and enable more robust corrective process control actions. The CPMS provides a novel way for controlling the cutting chip size and monitoring the surface roughness in milling processes through internet applications.

One cutting experiment with variable axial milling depth has been carried out to test the feasibility of the CPMS for surface roughness monitoring in endmilling. The results indicate the following:

- The proposed system, where the cloud computing resources and services are linked with the machine tool via hyper text transfer protocol, is feasible and could be efficiently implemented to monitor surface roughness during milling operation.

- The applied cloud based CNCS is stable which is reflected in improved surface quality.

- The application for cognitive corrective control acting manages to return the CSA to the reference chip size within $0.48 \mathrm{~s}$.

- The corrective control actions are taken within $10 \mathrm{~ms}$ after the significant change of the CSA is identified.

- The optical cutting chip size detecting was successfully used to control the machined $R a$.

- Controlled $R a$ does not deviate from the desired value for more than $10 \%$.

- The $R a$ is kept constant by regulating the CSA.

- The CPMS performs the $R a$ monitoring loop every $0.07 \mathrm{~s}$.

- The uncertainty associated with the prediction of surface roughness can be characterized as small, hence the ANFIS could properly predict the $R a$. Uncertainty analysis demonstrated that $98.2 \%$ of predicted values fall within the $95 \%$ confidence intervals. ANFIS in $1.8 \%$ of cases underestimated the values of $R a$ at small feed rates and at small chip sizes. The extent of these intervals with respect to the observed values was $92 \%$.

The research limitations of the introduced concept are summarized from the viewpoint of cloud computing, chip size acquiring and ANFIS based modelling. The limitations are given as follows:

- Massive amount of data to be processed. To perform the real-time monitoring a large amount of data, due to a measurement technique with high sampling rate is required for cyber machining. The impact of this limitation on the monitoring results can be minimized by reasonable selection of sensor type to achieve optimal ratio between data resolution and data processing quality and by deliberate selection of computing services to ensure the quality of data analysis.

- Cyber security and privacy limitation. Since sensing data and information for monitoring and corrective process control acting are no longer stored on the local pc-terminal, security and privacy issues become more serious in the CPS. A possible solution to protect the security of devices connected in the CPMS requires more advanced network traffic encryption technique such as secure socket layer and the transport layer for security.

- Limited data transfer speed. The speed of Internet connection limits the data transfer speed and affects successive data analysis performed by applications in the cloud. A solution to this problem is to perform preliminary signal preprocessing at local personal computer terminal and to extract features with compact data size, which are then transferred to the cloud for further analysis.

- Optimal illumination in visual system. For the future chip size detection system, it is still a challenge to control the adjustment of optimal illumination, which is a decisive factor for the quality of the images captured by a visual system.

- Wet machining. The proposed CPS can not be applied for wet machining, since the visual system of cutting chip size detecting is not efficient in wet conditions.

- Amount of data for modelling. The surface roughness model has to be built for each specific cutting tool geometry and tool diameter. Therefore, an extensive experimental testing has to be performed. However, the advantage of the 
ANFIS method is that the model can be trained and extended in real time during machining.

The special interest of future activities is to perform more tests with different cutting conditions in order to validate the proposed concept. Future work will be also directed to supplement the cloud platform with IoT applications for energy consumption monitoring and tool condition monitoring.

\section{REFERENCES}

[1] Wang, L., Törngren, M., Onori, M. (2015). Current status and advancement of cyber-physical systems in manufacturing. Journal of Manufacturing Systems, vol. 37, p. 517-527, D0I:10.1016/J.jmsy.2015.04.008.

[2] Wang, C., Ghani, S.B., Cheng, K., Rakowski, R. (2013). Adaptive smart machining based on using constant cutting force and a smart cutting tool. Proceedings of the Institution of Mechanical Engineers, Part B: Journal of Engineering Manufacture, vol. 227, no. 2, p. 249-253, DOl:10.1177/0954405412466233.

[3] Teti, R., Jemielniak, K., O'Donnell, G., Dornfeld, D. (2010). Advanced monitoring of machining operations. CIRP AnnalsManufacturing Technology, vol. 59, no. 2, p. 717-739, DOI:10.1016/j.cirp.2010.05.010.

[4] Chen, J.C., Huang, B. (2003). An in-process neural networkbased surface roughness prediction (INN-SRP) system using a dynamometer in end milling operations. The International Journal of Advanced Manufacturing Technology, vol. 21, no. 5, p. 339-347, DOl:10.1007/s001700300039.

[5] Abburi, N.R., Dixit, U.S. (2006). A knowledge-based system for the prediction of surface roughness in turning process. Robotics and Computer-Integrated Manufacturing, vol. 22, no. 4, p. 363-372, D0I:10.1016/j.rcim.2005.08.002.

[6] Rao, K.V., Murthy, B.S.N., Rao, N.M. (2013). Cutting tool condition monitoring by analyzing surface roughness, work piece vibration and volume of metal removed for AISI 1040 steel in boring. Measurement, vol. 46, no. 10, p. 4075-4084, D0I:10.1016/j.measurement.2013.07.021.

[7] Oborski, P. (2014). Developments in integration of advanced monitoring systems. The International Journal of Advanced Manufacturing Technology, vol. 75, no. 9-12, p. 1613-1632. D0l:10.1007/s00170-014-6123-x.

[8] Marinescu, I., Axinte, D.A. (2011). An automated monitoring solution for avoiding an increased number of surface anomalies during milling of aerospace alloys. International Journal of Machine Tools and Manufacture, vol. 51, no. 4, p. 349-357, D0I:10.1016/j.jimachtools.2010.10.005.
[9] Axinte, D., Boud, F., Penny, J., Gindy, N., Williams, D.J. (2005). Broaching of Ti-6-4-Detection of workpiece surface anomalies on dovetail slots through process monitoring. CIRP Annals - Manufacturing Technology, vol. 54, no. 1, p. 87-90, D0I:10.1016/S0007-8506(07)60056-0.

[10] Yang, L.-D., Chen, J.C., Chow, H.-M., Lin, C.-T. (2006). Fuzzynets-based in-process surface roughness adaptive control system in end-milling operations. The International Journal of Advanced Manufacturing Technology, vol. 28, no. 3-4, p. 236248, D0I:10.1007/s00170-004-2361-7.

[11] Zhang, J.Z., Chen, J.C. (2007). The development of an inprocess surface roughness adaptive control system in end milling operations. The International Journal of Advanced Manufacturing Technology, vol. 31, no. 9-10, p. 877-887, DOl:10.1007/s00170-005-0262-z.

[12] Zuperl, U., Cus, F. (2012). System for off-line feedrate optimization and neural force control in end milling. International Journal of Adaptive Control and Signal Processing, vol. 26, no. 2, p. 105-123, Dol:10.1002/acs.1277.

[13] Čuš, F., Župerl, U. (2015). Surface roughness control simulation of turning processes. Strojniški vestnik - Journal of Mechanical Engineering, vol. 61, no. 4, p. 245-253, DOI:10.5545/sv-jme.2014.2345.

[14] Zuperl, U., Cus, F. (2015). Simulation and visual control of chip size for constant surface roughness. International Journal of Simulation Modelling, vol. 14, no. 3, p. 392-403, DOI:10.2507/IJSIMM14(3)2.282.

[15] Shaw, M.C., Cookson, J.0. (2005). Metal Cutting Principles (vol. 2), Oxford university press, New York.

[16] Fenton, R.G., Oxley, P.L.B. (1969). Mechanics of orthogonal machining: predicting chip geometry and cutting forces from work-material properties and cutting conditions. Proceedings of the Institution of Mechanical Engineers, vol. 184, no. 1, p. 927-942, D0I:10.1243/PIME_PROC_1969_184_068_02.

[17] Dargusch, M.S., Sun, S., Kim, J.W., Li, T., Trimby, P., Cairney, J. (2018). Effect of tool wear evolution on chip formation during dry machining of Ti-6Al-4V alloy. International Journal of Machine Tools and Manufacture, vol. 126, p. 13-17, DOI:10.1016/j.ijmachtools.2017.12.003.

[18] List, G., Sutter, G., Bouthiche, A. (2012). Cutting temperature prediction in high speed machining by numerical modelling of chip formation and its dependence with crater wear. International Journal of Machine Tools and Manufacture, vol. 54-55, p. 1-9, D0I:10.1016/j.ijmachtools.2011.11.009.

[19] Marcé, R., Comerma, M., García, J.C., Armengol, J. (2004). A neuro-fuzzy modeling tool to estimate fluvial nutrient loads in watersheds under time-varying human impact. Limnology and Oceanography: Methods, vol. 2, no. 11, p. 342-355, DOI:10.4319/lom.2004.2.342. 